\title{
Cosmology Without Averaging
}

\section{Timothy Clifton}

Department of Astrophysics, Keble Road, University of Oxford, UK

and

Theoretical Physics Group, CERN, Geneva, Switzerland.

E-mail: t.clifton@cantab.net

PACS numbers: $98.80 . J \mathrm{k}$

\begin{abstract}
.
We construct cosmological models consisting of large numbers of identical, regularly spaced masses. These models do not rely on any averaging procedures, or on the existence of a global Friedmann-Robertson-Walker (FRW) background. They are solutions of Einstein's equations up to higher order corrections in a perturbative expansion, and have large-scale dynamics that are well modelled by the Friedmann equation. We find that the existence of arbitrarily large density contrasts does not change either the magnitude or scale of the background expansion, at least when masses are regularly arranged, and up to the prescribed level of accuracy. We also find that while the local space-time geometry inside each cell can be described as linearly perturbed FRW, one could argue that a more natural description is that of perturbed Minkowski space (in which case the scalar perturbations are simply Newtonian potentials). We expect these models to be of use for understanding and testing ideas about averaging in cosmology, as well as clarifying the relationship between global cosmological dynamics and the static space-times associated with isolated masses.
\end{abstract}




\section{Introduction}

The Friedmann-Robertson-Walker (FRW) model is ubiquitous in modern cosmology, and is widely believed to be a good model for a universe with a matter content that is approximately uniformly distributed over large scales. FRW has had great success in fitting a variety of cosmological observations, but is not without its problems. In particular, it has the apparent defect of requiring large amounts of dark matter and dark energy in order to be compatible with observations. More fundamentally, it relies on the implicit assumption that it is permissible to use non-local 'average' energy densities in Einstein's equations, which are a set of local field equations. The difficulties involved with this are exacerbated by the diffeomorphism covariance of the theory, which means that there is in general no preferred set of space-like hypersurfaces with which to perform an average over at all. What is more, even if a suitable and unique averaging scheme is found, it seems we will still be left with a back-reaction effect due to the non-commutativity of averaging and evolution under Einstein's equations [1, 2]. These issues require further study in order to be fully understood.

One way to make progress in this area is to construct alternative models that are approximately homogeneous and isotropic on the largest scales, but that do not involve any averaging procedures. We will survey some of the relevant literature on progress toward this goal in the section that follows, and then proceed to present our own approach to constructing a cosmological model that is composed of discrete masses, rather than a continuous fluid. This model will appear homogeneous and isotropic when coarse-grained over the largest scales, but will also be able to accommodate arbitrarily large local density contrasts. The validity of this model will be based solely on Newtonian and post-Newtonian perturbative expansions about Minkowski space, which are generally considered to be able to easily model large density gradients without breaking down (for a recent discussion of the potential difficulties involved in modelling the corresponding situation with a perfect fluid filled, perturbed FRW cosmology see, e.g., [3]).

The model we construct is one in which isolated masses are arranged in a regular array, which by application of the Israel junction conditions is a solution of Einstein's equations up to a specified level of accuracy. Contrary to some previous results in the literature, we show that discretisation of the matter content does not affect the global expansion of the space-time, which proceeds in just the same way as a perfect fluid FRW solution with the same energy density and spatial curvature, up to the required accuracy. This result says nothing about what happens in the more realistic situation of irregularly arranged masses, but does show that, in at least some situations, perturbed FRW is a good description of a universe with (arbitrarily) large density contrasts. Establishing the existence of such situations is of interest not only for limiting the possible consequences of inhomogeneity on the large-scale expansion of the Universe, but also for testing the viability of proposed methods of accounting for more general inhomogeneity in the Universe: If they predict modifications to the large-scale expansion in configurations 
where it is known that none should occur, then one may choose to question their viability.

In Section 2 we survey some of the literature on inhomogeneous cosmological models, concentrating in particular on studies that have similarities to the approach used here, and highlighting predictions of deviations from the usual Friedmann expansion. In Section 3 we present the type of model we will be considering, and the perturbative expansion we deploy is outlined in Section 4. Section 5 treats the geometry inside each of the primitive cells of our structure as being perturbed Minkowski space, and finds the corresponding cosmological evolution, after the Israel junction conditions have been applied. A similar analysis is then performed in Section 6, this time around a time dependent background. In Section 7 we relate the time independent and time dependent approaches used in the preceding two sections, and in Section 8 we show that the model we have constructed has identical large-scale expansion to a perfect fluid filled FRW cosmology with the same energy density and large-scale spatial curvature. In Section 9 we consider the problem of determining cosmological observables, such as redshifts and luminosity distances, and in Section 10 we conclude.

\section{Previous Results}

Before proceeding with our study, let us briefly review some of the relevant literature on cosmological models containing discrete masses, and the affect of structure on the evolution of the Universe. These studies have frequently suggested that the presence of inhomogeneity in the Universe could affect its global expansion rate.

One of the first studies to include discrete masses in a cosmological model was that of McVittie [4]. Here a space-time geometry similar to the Einstein static universe was considered, and McVittie concluded that if instead of having a perfectly homogeneous and isotropic matter content, it was instead the case that a number, $n$, of singularities of mass $m$ were allowed to develop, then the corresponding spatial volume of the universe would be

$$
V=\frac{16 M^{3}}{\pi}\left(1+\frac{2 n m}{M}\right)>\frac{16 M^{3}}{\pi}=V_{\text {Einstein }},
$$

where $M$ is the total mass in the universe, and $V_{\text {Einstein }}$ is the spatial volume of the perfect Einstein static universe. McVittie then reasoned that because the spatial volume of such a universe was larger after structures were allowed to form, that this signalled instability of the Einstein static universe, which requires a particular value of $V$ in order to exist. For the present purposes one could interpret this result as saying that the presence of discrete structures changes the scale of the cosmological solution.

The next significant development we are aware of, in terms of cosmological models containing discrete structures, is due to Einstein and Strauss [5]. Here the authors embed discrete masses into an FRW background by excising spherical regions of the homogeneous fluid, and replacing them with vacuoles containing singularities at their centres. The space-time inside each 'vacuole' is then well modelled by either Schwarzschild geometry, or perturbed FRW (provided the vacuole is small compared to 


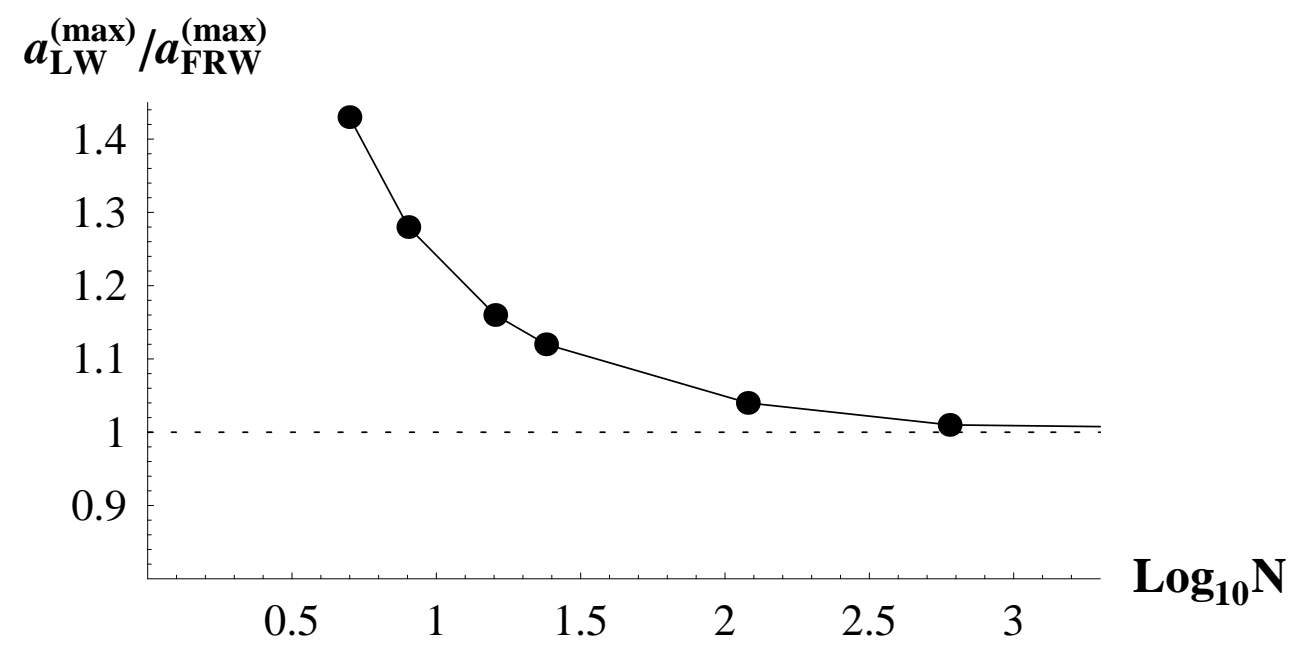

Figure 1. The maximum of expansion in the Lindquist-Wheeler model, $a_{L W}^{(\max )}$, as a fraction of the corresponding maximum in a closed FRW universe with the same energy density and spatial curvature, $a_{F R W}^{(\max )}$. The abscissa gives the number of cells in the Lindquist-Wheeler lattice, $N$.

the Hubble scale of the background solution). The resulting structure is often referred to as a 'Swiss cheese universe', and is an exact solution of Einstein's equations when Schwarzschild geometry is used. In these models the presence of the singularities does not affect the expansion of the background FRW space-time. One should, however, be aware that this is true by construction: The vacuoles all have exactly FRW boundary conditions, and are not allowed to intersect in the usual application of the model. Furthermore, one could question whether the requirement of spherical symmetry restricts the possible behaviours that might otherwise be possible. In the present study we will lift the requirements of perfect FRW boundary conditions and spherical symmetry, although the models we consider are still highly symmetric.

In 1957 the problem of cosmological models containing discrete masses was returned to by Richard Lindquist and John Archibald Wheeler [6]. These authors attempted to build a cosmological model in analogy to the Wigner-Seitz construction of solid-state physics. The basic idea here is to construct a regular lattice of cells, and then solve the field equations by approximating the influence of all cells external to the one under consideration as being spherically symmetric. In the context of general relativity this results in the space-time inside each cell being uniquely given by Schwarzschild geometry, and unlike the case of solid-state physics, results in a non-zero normal derivative of the relevant field at the boundary of each cell. The lattice therefore undergoes a global expansion, that can be compared to the Friedmann solutions of FRW cosmology. By considering spatially closed lattices Lindquist and Wheeler deduced that the expansion of their model had the same functional form as the usual Friedmann solution, but with a different scale of expansion, as shown in Fig. 1. Luminosity distances and redshifts in this model were calculated in [7] and [8], where deviations from the corresponding quantities in FRW cosmology were identified. The problem of finding an exact 2- 
body solution within this framework has also been addressed recently in [9]. While compelling, however, the Lindquist-Wheeler model suffers from the problem of relying on an approximation scheme that is difficult to quantify. In the present study we attempt to produce a similar model to that created by Lindquist and Wheeler, but with a more clearly defined set of approximations.

More recent studies on the affect of structure on the expansion of the Universe often come under the title 'back-reaction', or 'macroscopic gravity'. Let us consider the case of macroscopic gravity first. The basic idea behind this approach is that when describing an 'average' cosmological evolution we are not necessarily interested in the local expansion rate any any particular point in the Universe, but rather in the non-local expansion inferred from observations made over large distances. Now, while Einstein's equations are thought to be the appropriate ones to describe the local curvature of space-time, if we consider non-local quantities, such as 'average' expansion, then we may need a different set of equations. The problem of how averaging should best be performed in general relativity is a difficult, and still somewhat open question. Progress toward solving this problem has, however, been made by Zalaletdinov in his macroscopic gravity theory, which modifies Einstein's equations by including gravitational correlation correction terms [10]. Applying this approach to cosmological solutions Coley, Pelavas and Zalaletdinov have found that there exist homogeneous and isotropic exact solutions to the macroscopic field equations in which the correlation tensor takes the form of a spatial curvature term [11]. This result again supports the idea that the formation of structure in the Universe could lead to a change in scale of the global expansion.

Finally, let us return to studies of the 'back-reaction' problem. The basic idea here is that averaging over a space-like hyper-surface, and evolution of the same hyper-surface under Einstein's equations, do not commute, so that if we wish to successfully evolve an averaged space forward in time we should add corrections to Einstein's equations. The affect of these extra terms is what is known as the back-reaction of the structures that form in the Universe on the overall expansion, and is a subject that has recently attracted much attention (see, e.g., [12] for a review and references). It was shown in [13] that for scaling solutions to exist in this approach then the extra terms in the effective Friedmann equation should either appear as an effective massless scalar field, or with the same form as the spatial curvature term. The former case is inconsequential for the expansion of the late Universe, but if the latter is true then this once again points to a change in scale of the global cosmological dynamics (although one should be aware that the spatial curvature term in the effective Friedmann equation here does not have to scale as it usually does in FRW cosmology).

We consider the results of these previous investigations more than sufficient motivation to further investigate models with a discretized matter content. 


\section{A Lattice of Discrete Masses}

Our aim is to further develop the ideas outlined above by constructing a well defined cosmological model in which the Universe is filled with a large number of identical, regularly spaced masses, but has no fluid filled background cosmology. To achieve this we begin as Lindquist and Wheeler did, by considering a number of cells that are regular polytopes, and that are identical to each another up to spatial translations and rotations. At the centre of each cell we then place a non-rotating, chargeless object of mass $m$. These cells will act as the building blocks of our model, and our objective is to arrange them in such a way as to construct a smooth, geodesically complete space-timet.

To satisfy Einstein's equations we must have that the geometry inside each cell satisfies $R_{a b}=0$ in the exterior region of the central mass, and that the boundaries between cells satisfy the Israel junction conditions in vacuum: That the induced metric and extrinsic curvature of the boundary are the same on either side [14, 15]. The high degree of symmetry in the situation we are considering makes these conditions considerably simpler than they are in general. Mirror symmetry of any two cells about their mutual boundary means that the induced metrics on either side of that boundary are automatically identical. The conditions of identical extrinsic curvature are less trivial, but the symmetry of the situation is again very useful.

In Figure 2 we show a cross-section of the two cells we are trying to match in some coordinates $x^{a}$ for the first cell, and $x^{\hat{a}}$ for the second cell. The extrinsic curvature in the first cell is then given by $K_{a b}^{(1)}=n_{a ; b}^{(1)}$, where $n_{a}^{(1)}$ is the space-like unit vector normal to the boundary, and pointing out of the cell. In the second cell, the extrinsic curvature is similarly given as $K_{\hat{a} \hat{b}}^{(2)}=n_{\hat{a} ; \hat{b}}^{(2)}$, where $n_{\hat{a}}^{(2)}$ is again the space-like unit vector normal to the boundary, but this time pointing into the cell. The covariant derivatives in these expressions should be taken with respect to the space-time geometry of the cell in question. The conditions for identical extrinsic curvature of the boundary on either side are then

$$
\frac{\partial x^{a}}{\partial \xi^{i}} \frac{\partial x^{b}}{\partial \xi^{j}} K_{a b}^{(1)}=\frac{\partial x^{\hat{a}}}{\partial \xi^{i}} \frac{\partial x^{\hat{b}}}{\partial \xi^{j}} K_{\hat{a} \hat{b}}^{(2)} \equiv K_{i j},
$$

where $\xi^{i}$ are intrinsic coordinates on the boundary. From the symmetry of the situation, however, it can be seen that we could just as easily have calculated the extrinsic curvature of the boundary in the second cell by taking the covariant derivative of an inward pointing normal vector in the first cell, as illustrated in Figure 2, As this vector is given by $-n_{a}^{(1)}$ we must have $K_{i j}=-K_{i j}$ in order to satisfy (2), or equivalently

$$
K_{i j}=0 \text {. }
$$

The junction conditions are therefore satisfied if, and only if, the $2+1$ dimensional boundary is extrinsically flat.

Now that boundary conditions for each of the cells are known, the field equations within each cell can be solved. These are simply the vacuum Einstein equations, $R_{a b}=0$.

$\ddagger$ Up to the possible existence of singularities at the centre of each cell. 

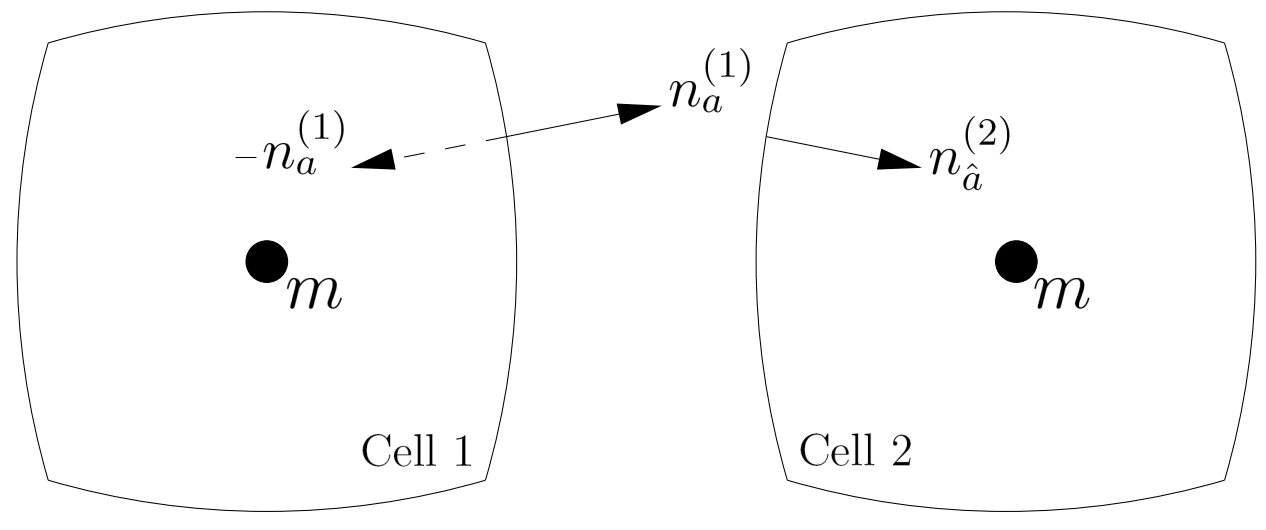

Figure 2. A schematic of the vectors involved in matching two cubic cells with central mass $m . n_{a}^{(1)}$ and $n_{\hat{a}}^{(2)}$ are space-like unit vectors normal to the boundary. The dashed vector is $-n_{a}^{(1)}$, and is mirror symmetric with $n_{\hat{a}}^{(2)}$.

Note, however, that without boundary conditions these equations do not have a unique solution. We therefore need Eq. (3) to find the space-time geometry inside each cell. As we will discuss in the next section, we expect this space-time to be well described by the usual Newtonian and post-Newtonian approach. This does not, however, mean that the entire cosmological model can be described in a single Newtonian frame-work: The Newtonian descriptions valid within each cell cannot describe arbitrarily large numbers of cells simultaneously. This should be clear from the fact that on scales of the order of a Hubble length we expect recessional velocities to approach the speed of light. Rather, the way in which the Newtonian descriptions that are valid within the domain of each individual cell should be related to one another can be deduced from the boundary conditions, Eq. (3) , and will be spelled out in the sections that follow.

Unlike most approaches to building a cosmological mode, the current one does not require us to write down one line-element that is valid for the entire observable Universe. Instead, due to the periodicity of the structure we are considering, it is sufficient to consider only a single cell. Once we know the geometry, extent, and rate of expansion of any one cell, we then know the space-time geometry of the entire universe (up to regions were our approximations break down, as should be expected, for example, in the early universe, or near the Schwarzschild radius of a compact object).

One could also, conceivably, consider more complicated structures than the simple polychora described above. As long as it can be shown that the space-time geometry is symmetric about the boundaries between cells, then the junction conditions will still be satisfied by Eq. (3), and the global space-time geometry can again be deduced in the manner just described. In such a case, however, there may be more than a single type of primitive cell to consider. We will not try and construct such situations here, preferring instead to concentrate on the simplest structures possible: Those built from a single repeated polytope. 


\section{Perturbative Expansion}

We will not attempt to proceed by looking for an exact solution to Einstein's equations, as we expect this would be prohibitively difficult. Instead we will treat the problem perturbatively, and within each cell will expand with respect to some small parameter:

$$
\epsilon \sim \frac{v}{c},
$$

where $v$ is the typical velocity associated with the type of objects we will be considering, and $c$ is the speed of light. For planetary and galactic systems it is almost always the case that $v / c \lesssim 0.01$. Furthermore the Newtonian potentials associated with such systems are nowhere greater than $\phi \sim 10^{-4}$, except within the vicinity of black holes and neutron stars. We can therefore assign

$$
\phi \sim \epsilon^{2} .
$$

Given that the evolution of these systems are governed by the motion of their constituents we also have that $\partial / \partial t \sim v \cdot \nabla$, which implies that

$$
\frac{|\partial / \partial t|}{|\partial / \partial x|} \sim \epsilon
$$

so that time derivatives add an extra order of smallness. Booking in orders of smallness in this way is familiar from the usual approach to post-Newtonian gravitational physics [16]. Here, however, we will be concerned with the vacuum region outside of the central mass of each of our cells. We therefore need not consider the orders of smallness associated with rest mass density, pressure, or any other form of energy density.

We expect the expansion we have just described to be applicable as long as the size of each cell is large compared with the Schwarzschild radius of its central mass, and as long as the number of cells within one cosmological horizon is also large. That is, we will be considering situations in which the bulk of the interior of each cell is well described by the usual Newtonian and post-Newtonian gravitational physics. We will not be concerning ourselves here with what happens near the singularities that may exist at the centre of each cell, and will not allow the cells to be so large that their boundaries would appear to have a recessional velocity any greater than $\sim 0.01 c$. In fact, we will have in mind throughout this article cells that are about $1 \mathrm{Mpc}$ wide, with a Milky Way sized mass at their centre, so that $\phi \sim 10^{-7}$ at the edge of each cell. This is well within the limits just mentioned. For further details of the perturbative expansion used in post-Newtonian physics we refer the reader to [16].

Let us now consider specifically the motion of our cell boundaries, whose trajectory we will take to be tangent to the 4 -vector $X^{a}$. If $n_{a}$ is normal to this boundary then we can write that the boundary has 4 -velocity

$$
u^{a} \equiv \frac{d X^{a}}{d \tau}=\frac{d t}{d \tau}\left(1 ; \frac{d X^{\mu}}{d t}\right),
$$

where $\tau$ is proper time along $X^{a}$, and $\mu$ runs over spatial indices, and that the condition $u^{a} n_{a}=0$ then gives (for $d t / d \tau \neq 0$ )

$$
n_{t}=-n_{\mu} \frac{d X^{\mu}}{d t} .
$$


Hence, if $d X^{\mu} / d t \sim O(\epsilon)$, so that it has the order of magnitude associated with a velocity in the perturbative expansion just outlined, then

$$
\frac{n_{t}}{n_{\mu}} \sim \epsilon .
$$

It then follows that $n_{t}$ has an $O(\epsilon)$ of smallness compared to $n_{\mu}$, which is expected to be $\sim 1$. Rather than explicitly quoting $\epsilon$ in what follows, we will instead simply state that quantities have a certain order of smallness associated with them. That this smallness is prescribed by a factor of $\epsilon$ should be taken to be implicit.

\section{Fluctuations About a Static Background}

\subsection{Large-scale Evolution Equations}

We can now address the question of the space-time geometry inside of each cell, and the motion of the cell boundaries that results from Eq. (3). Our initial ansatz for the geometry inside a cell will be linear perturbations around a Minkowski background. This is the standard way to model the weak gravitational fields around massive objects. For this, we will use the 'conformal Newtonian' gauge, where the line-element is written

$$
d s^{2}=-(1+2 \phi) d t^{2}+(1-2 \psi)\left(d x^{2}+d y^{2}+d z^{2}\right) .
$$

Here the functions $\phi$ and $\psi$ are gravitational potentials, and in general relativity we have the well known result that $\psi=\phi$.

Using the perturbative expansion outlined in Section 4, we can now write the lowest non-trivial order of each component of the extrinsic curvature of the boundary at the edge of one of our cells as

$$
\begin{aligned}
K_{a b} d x^{a} d x^{b}= & \left(n_{t, t}-n_{\mu} \phi_{, \mu}\right) d t^{2}+\left(n_{\mu, t}+n_{t, \mu}\right) d x^{\mu} d t \\
& +\left(n_{\mu, \nu}+2 \psi_{, \mu} n_{\nu}-\delta_{\mu, \nu} \psi_{, \sigma} n_{\sigma}\right) d x^{\mu} d x^{\nu},
\end{aligned}
$$

where indices $\mu, \nu, \sigma$ denote spatial components. The $K_{t t}$ and $K_{\mu \nu}$ terms should be expected to have $O(4)$ corrections here, and the $K_{t \mu}$ and $K_{\mu t}$ terms $O(3)$ corrections. The time component of the unit vector normal to $\Sigma$ has been assigned an $O(1)$ of smallness in comparison to the space-like components here, as discussed in Section 4 .

Now, let us apply the coordinate transformation from Eq. (21) to the expression in Eq. (11), in order to find $K_{i j}$. To explicitly give the coordinates $\xi^{i}$ required for this let us first pick out a preferred space-like direction $x$, which is orthogonal to the boundary at point where it is at its closest to the central mass. Such a direction can always be made to correspond to one of the coordinates in Eq. (10) by performing spatial rotations. The remaining two spatial directions are then uniquely defined up to a rotation, and we will denote these directions by the indices $A, B, C$ etc.. We can now choose coordinates on the boundary at $x=X\left(t, x^{A}\right)$ to be given by $\xi^{i}=\left(t, x^{A}\right)$. Finally, let us define two new

derivative operators which act along the boundary in time-like and space-like directions:

$$
\begin{gathered}
\cdot \equiv u^{a} \partial_{a}=\partial_{t}+X_{, t} \partial_{x} \\
\mid A \equiv m^{a} \partial_{a}=\partial_{A}+X_{, A} \partial_{x},
\end{gathered}
$$


where $u^{a}$ and $m^{a}$ are time-like and space-like vectors in the boundary in planes of constant $x^{A}$ and $t$, respectively. The extrinsic curvature of the boundary can then be written in the coordinate basis of $\xi^{i}$ as

$$
\begin{aligned}
K_{i j} d \xi^{i} d \xi^{j}= & \left(\dot{n}_{t}+\dot{X} \dot{n}_{x}-n_{\mu} \phi_{, \mu}\right) d t^{2}+\left(\dot{n}_{A}+n_{t \mid A}+X_{\mid A} \dot{n}_{x}+\dot{X} n_{x \mid A}\right) d x^{A} d t \\
+ & {\left[n_{A \mid B}+X_{\mid A} n_{x \mid B}-\left(\psi_{, C} n_{C}-\psi_{, x} n_{x}\right)\left(\delta_{A B}+X_{\mid A} X_{\mid B}\right)\right.} \\
& \left.\quad-2\left(\delta_{A B} \psi_{, x} n_{x}-\psi_{, A} n_{B}-\psi_{, x} X_{\mid A} n_{B}-n_{x} \psi_{, A} X_{\mid B}\right)\right] d x^{A} d x^{B}
\end{aligned}
$$

where space-time dependent quantities, such as derivatives of $\phi$ and $\psi$, should implicitly be taken to be evaluated on the boundary. This expression can now be simplified by making use of the orthogonality of $n^{a}$ with respect to $u^{a}$ and $m^{a}$. This gives us

$$
\begin{aligned}
& n_{t}=-n_{x} X_{, t} \\
& n_{A}=-n_{x} X_{, A},
\end{aligned}
$$

which allows us to write Eq. (12) as

$$
\begin{aligned}
K_{i j} d \xi^{i} d \xi^{j}=-n_{x}[ & \left(\ddot{X}+\phi_{, x}-X_{\mid A} \phi_{, A}\right) d t^{2}+\left(\left(X_{\mid A}\right)^{\cdot}+(\dot{X})_{\mid A}\right) d x^{A} d t \\
& \left.+\left(X_{\mid A B}+\left(\psi_{, x}-X_{\mid C} \psi_{\mid C}\right)\left(\delta_{A B}+X_{\mid A} X_{\mid B}\right)\right) d x^{A} d x^{B}\right] .
\end{aligned}
$$

The boundary conditions in Eq. (3) can now be straight-forwardly applied to (15).

We find that our lattice is a solution of Einstein's equations (to lowest order in our perturbative expansion) if the boundary satisfies the conditions:

$$
\ddot{X}=-\left.\sqrt{1+\left(X_{\mid A}\right)^{2}}(n \cdot \nabla \phi)\right|_{X}+O(4)
$$

and

$$
X_{\mid A B}=\left(\delta_{A B}+X_{\mid A} X_{\mid B}\right) \ddot{X}+O(4)
$$

together with $\left(X_{\mid A}\right)^{\cdot}=(\dot{X})_{\mid A}=0+O(3)$, where we have now taken $\psi=\phi$, and where we have used $n^{a} n_{a}=1$. On the RHS of these equations we have also used the notation $\nabla=\partial_{\mu}$, and made explicit that the gradient of $\phi$ should be evaluated at $x=X$.

The potential $\phi$ must of course also satisfy the field equation $R_{a b}=0$ in the bulk of the cell, which gives us the equations:

$$
\nabla^{2} \phi=0+O(4)
$$

and

$$
(\nabla \phi)_{, t}=0+O(5) .
$$

If Eqs. (16)-(19) are satisfied, then our lattice is a cosmological solution to Einstein's equations, up to the specified order, with arbitrarily large density contrast, and without any averaging having been performed. 


\subsection{Cosmological Solutions}

Above we have derived the evolution equations for a lattice constructed from a large number of individual cells, each of which contains an isolated central mass. In this case the metric fluctuations $\phi$ can be seen to be given simply by the usual solutions to the Newtonian Poisson equation in vacuum, with Neumann boundary conditions specified on $X$ via Eq. (16). One can then see from Eq. (16) that if $\left.(n \cdot \nabla \phi)\right|_{X}>0$ (so that the force due to the central mass is attractive) then the boundaries must follow trajectories along which $\ddot{X}<0$. The global expansion must therefore always be decelerating. Eq. (17) then tells us that we must also have $X_{\mid y y}$ and $X_{\mid z z}<0$, so that in the coordinates of Eq. (10) the boundaries are concave, when viewed from inside the cell (as is supposed to be implied in Figure 21).

The first thing that one can now find is the equation of motion of the element of the boundary that is closest to the central mass of the cell. We will label the position of this closest point as $X_{c}(t)=X(t, 0,0)$. From our choice of coordinate we then have $X_{c \mid y}=X_{c \mid z}=0$ and $\left.(n \cdot \nabla \phi)\right|_{X_{c}}=\left.\phi_{, x}\right|_{X_{c}}$, so that Eq. (16) becomes

$$
\ddot{X}_{c}=-\left.\phi_{, x}\right|_{X_{c}}+O(4) \text {. }
$$

This boundary element is therefore in free fall in the potential $\phi$, and the shape of the boundary at this point is given simply by $X_{c \mid y y}=X_{c \mid z z}=-\left.\phi_{, x}\right|_{X_{c}}$. Given Eq. (20) for $X_{c}$, we can now see that the solution for $X$ at all other $y$ and $z$ is given by

$$
X=X_{c}+\frac{1}{2} \ddot{X}_{c}\left(y^{2}+z^{2}\right)+O(4) .
$$

Direct substitution of this expression into Eq. (16) and (17) shows it to be a solution, up to the required order, as long as $y$ and $z$ are always small compared to $\sim \dot{X}^{-1}$. This condition should always be true as long as each individual cell is small compared to the Hubble scale of the lattice, which is the situation we outlined to begin with.

Now, if $\phi$ is a solution to the Newtonian Laplace equation, (18), then one may expect the solutions of Eq. (20) to obey the classification scheme of the usual Newtonian $n$ body problem given by Saari [17. Roughly speaking, this corresponds to the following: If $d X / d t$ is large enough then $X \rightarrow t$ at late-times, otherwise $X \rightarrow t^{2 / 3}$ or we have eventual recollapse. More directly, at some time $t=t_{0}$ one can place an initial boundary at $x=X\left(t_{0}, y, z\right)$ and give it some initial velocity $\dot{X}\left(t_{0}, y, z\right)$. Eqs. (16) and (17) then tell us what $\left.\phi_{, x}\right|_{X}$ and $\ddot{X}$ are along the boundary at $t=t_{0}$. This is enough information to evolve the boundary forward in time, obtaining $X$ and $\left.\phi_{, x}\right|_{X}$ at every $t$, and for every $y$ and $z$. It is therefore also sufficient to provide the necessary Neumann boundary conditions with which we can solve Eq. (18) for $\phi\left(x^{\mu}\right)$ at every $t$. Of course, this can only be done in ways that satisfy Eq. (19), and the required properties of the solutions of Eq. (18). In particular, the 'maximum principle' of harmonic functions tells us that as long as gravity is attractive near the central mass then the boundary cannot accelerate. 


\section{Fluctuations About Time-dependent Backgrounds}

\subsection{Large-scale Evolution Equations}

We can also consider modelling the space-time inside each cell as fluctuations about time-dependent FRW backgrounds, such that the line-element can be written as

$$
d s^{2}=-(1+2 \Phi) d \hat{t}^{2}+\frac{a^{2}(1-2 \Psi)}{\left[1+\frac{k}{4}\left(\hat{x}^{2}+\hat{y}^{2}+\hat{z}^{2}\right)\right]^{2}}\left(d \hat{x}^{2}+d \hat{y}^{2}+d \hat{z}^{2}\right),
$$

where $a=a(\hat{t})$ and $k=$ constant. We are using hatted coordinates here, and capital $\Phi$ and $\Psi$, to distinguish these quantities from the coordinates and potentials used in the previous section.

Substituting Eq. (22) into the Einstein equations $R_{\hat{a} \hat{b}}=0$ gives us that $a$ and $\Phi$ must satisfy the following equations in the bulk of the cell

$$
\frac{a_{, \hat{t}}^{2}}{a^{2}}=-\frac{2 \hat{\nabla}^{2} \Phi}{3 a^{2}}-\frac{k}{a^{2}}+O(4) \quad \text { and } \quad \frac{a_{, \hat{t} \hat{t}}}{a}=\frac{\hat{\nabla}^{2} \Phi}{3 a^{2}}+O(4),
$$

where $(a \hat{\nabla} \Phi)_{\hat{t}}=0+O(3)$, where $\Psi=\Phi$ and where $\hat{\nabla}=\partial_{\hat{\mu}}$ is the spatial derivative operator in the hatted coordinate system. These are clearly just the Friedmann equations, with $\hat{\nabla}^{2} \Phi / a^{2} \propto 1 / a^{3}$ acting as a pressure-less dust term. If $\hat{\nabla}^{2} \Phi=0$, as in the previous section, then the background space-time is Milne. More generally $a(\hat{t})$ behaves as in an FRW universe with dust and spatial curvature. From Eq. (23) it can also be seen that we should assign to $k$ an $O\left(\epsilon^{2}\right)$ of smallnes:\$.

As before, let us again consider a cell boundary at $\hat{x}=\hat{X}(\hat{t}, \hat{y}, \hat{z})$. The extrinsic curvature of such a $2+1$ dimensional surface is now given, in the coordinates of Eq. (22), by

$$
\begin{aligned}
K_{\hat{a} \hat{b}} d x^{\hat{a}} d x^{\hat{b}}= & \left(n_{\hat{t}, \hat{t}}-\frac{1}{a^{2}} n_{\hat{\mu}} \Phi_{, \hat{\mu}}\right) d \hat{t}^{2}+\left(n_{\hat{\mu}, \hat{t}}+n_{\hat{t}, \hat{\mu}}-2 \frac{a_{, \hat{t}}}{a} n_{\hat{\mu}}\right) d x^{\hat{\mu}} d \hat{t} \\
& +\left[n_{\hat{\mu}, \hat{\nu}}+\left(k x^{\hat{\mu}}+2 \Psi_{, \hat{\mu}}\right) n_{\hat{\nu}}-\delta_{\hat{\mu} \hat{\nu}}\left(\left(\frac{1}{2} k x^{\hat{\sigma}}+\Psi_{, \hat{\sigma}}\right) n_{\hat{\sigma}}+a a_{, \hat{t}} n_{\hat{t}}\right)\right] d x^{\hat{\mu}} d x^{\hat{\nu}},
\end{aligned}
$$

where quantities with hatted coordinates should be taken to correspond to those associated with the time-dependent geometry given in Eq. (22). We can now proceed as in the previous case, making analogous definitions, to find the extrinsic curvature on a boundary at $\hat{x}=\hat{X}\left(\hat{t}, x^{\hat{A}}\right)$ to be

$$
\begin{aligned}
K_{\hat{i} \hat{j}} d \xi^{\hat{i}} d \xi^{\hat{j}}=-n_{\hat{x}}[ & \left(\ddot{\hat{X}}+2 \frac{\dot{a}}{a} \dot{X}+\frac{1}{a^{2}} \Phi_{, \hat{x}}-\frac{1}{a^{2}} \hat{X}_{\mid \hat{A}} \Phi_{, A}\right) d \hat{t}^{2} \\
& +\left(\left(\hat{X}_{\mid \hat{A}}\right)^{\cdot}+(\dot{\hat{X}})_{\mid \hat{A}}-2 \frac{\dot{a}}{a} \hat{X}_{\mid \hat{A}}\right) d x^{\hat{A}} d \hat{t} \\
& \left.+\left(\hat{X}_{\mid \hat{A} \hat{B}}-\mathcal{J}\left(\hat{t}, \hat{X}, x^{\hat{A}}\right)\left(\delta_{\hat{A} \hat{B}}+\hat{X}_{\mid \hat{A}} \hat{X}_{\mid \hat{B}}\right)\right) d x^{\hat{A}} d x^{\hat{B}}\right],
\end{aligned}
$$

$\S$ We could choose to rescale $k$ to 0 or \pm 1 , but in this case we would also have to rescale all other dimensionful quantities, so the overall perturbative expansion would remain unchanged. 
where we have defined

$$
\mathcal{J}\left(\hat{t}, \hat{X}, x^{\hat{A}}\right) \equiv a \dot{a} \dot{\hat{X}}-\Psi_{, \hat{x}}+\Psi_{, \hat{A}} \hat{X}_{\mid \hat{A}}-\frac{1}{2} k \hat{X}+\frac{1}{2} k x^{\hat{A}} \hat{X}_{\mid \hat{A}}
$$

The junction conditions given in Eq. (3) now tell us that the boundary must satisfy the following equations, to lowest order in our expansion:

$$
\frac{\ddot{\hat{X}}}{\hat{X}}=-2 \frac{\dot{a}}{a} \frac{\dot{\hat{X}}}{\hat{X}}-\frac{\Phi_{, \hat{x}}}{a^{2} \hat{X}}+\frac{\hat{X}_{\mid \hat{A}} \Phi_{, \hat{A}}}{a^{2} \hat{X}}+O(4)
$$

and

$$
\hat{X}_{\mid \hat{A} \hat{B}}=\mathcal{J}\left(\hat{t}, \hat{X}, x^{\hat{A}}\right)\left(\delta_{\hat{A} \hat{B}}+\hat{X}_{\mid \hat{A}} \hat{X}_{\mid \hat{B}}\right)+O(4)
$$

with $\left(\hat{X}_{\mid \hat{A}}\right)^{\cdot}=(\dot{\hat{X}})_{\mid \hat{A}}=2(\dot{a} / a) \hat{X}_{\mid \hat{A}}+O(3)$. These equations must be satisfied, together with the bulk field equations, (23) equations.

\subsection{Cosmological Solutions}

Eqs. (23), (27) and (28) admit as a solution a lattice cell with boundaries that are static in the coordinates of Eq. (22). In this case, $\hat{X}$ must satisfy

$$
\hat{X}_{\mid \hat{A} \hat{B}}=-\frac{k}{2}\left(\hat{X}-\hat{X}_{\mid \hat{C}} x^{\hat{C}}\right)\left(\delta_{\hat{A} \hat{B}}+X_{\mid \hat{A}} X_{\mid \hat{B}}\right)+O(4),
$$

for all $\hat{t}$, which has the solution

$$
\hat{X}=\hat{X}_{0}\left(1-\frac{k}{4}\left(\hat{y}^{2}+\hat{z}^{2}\right)\right)+O(4),
$$

where $\hat{X}_{0}$ is a constant. As $\hat{X}$ is not a function of $t$ here, the global expansion of the space-time is fully specified by $a(\hat{t})$ alone. Eq. (27) then tells us that for Eq. (29) to hold we must require

$$
\left.\hat{n} \cdot \hat{\nabla} \Phi\right|_{\hat{X}}=0+O(4)
$$

on the boundary. Now, it also clear from Eq. (23) that the potential $\Phi$ must satisfy

$$
a \hat{\nabla}^{2} \Phi=c_{1}+O(4)
$$

where $c_{1}$ is a constant in both $\hat{t}$ and $x^{\hat{\mu}}$, and is $O(2)$ in smallness. This equation has the solution

$$
\Phi=\frac{\Phi_{N}}{a}+\frac{c_{1}}{6 a}\left(\hat{x}^{2}+\hat{y}^{2}+\hat{z}^{2}\right)+O(4)
$$

where $\Phi_{N}=\Phi_{N}\left(x^{\hat{\mu}}\right)$ is the Newtonian potential that satisfies $\hat{\nabla}^{2} \Phi_{N}=0$. The extra nonNewtonian term must occur for all time-dependent backgrounds other than the Milne universe, and "appears due to the fact that in the present case we have no embedding in the Euclidean space" [5]. We will call this term the vacuum potential.

The solutions (29) and (33) above can be seen to satisfy the condition (31) if, and only if,

$$
c_{1}=-\left.\frac{3}{\hat{X}} \Phi_{N, x}\right|_{\hat{X}} .
$$


One can then see that if the Newtonian potential is attractive, so that $\Phi_{N, \hat{x}}>0$, then boundaries stay at fixed $\hat{X}$ if, and only if, $c_{1}<0$. It can also be seen from Eq. (30) that for $k=0$ a boundary at static $\hat{X}$ must be in a plane of $\hat{x}=$ constant, while for $k<0$ or $k>0$ the boundary must be either convex or concave, respectively, when viewed from inside the cell in the coordinates of Eq. (22). It is also clear from Eq. (23) that the functional form of $a(\hat{t})$ in each of these cases must be the same as in an FRW universe with the same $k$.

Let us also note that solutions with boundaries at static $\hat{X}$ require the forces from the Newtonian and vacuum potentials in Eq. (33) to be in unstable equilibrium. This is due to the force from the Newtonian potential being attractive and growing as $\hat{X}$ becomes smaller, while the force from the vacuum potential pushes the boundary to greater $\hat{X}$ (as long as $c_{1}<0$ ), and grows with $\hat{X}$. So, if the boundary should be perturbed to slightly large $\hat{X}$, then the vacuum potential should come to dominate, and cause $d^{2} \hat{X} / d \hat{t}^{2}>0$. This does not, however, correspond to acceleration in the more cosmologically relevant proper distance $R=a \hat{X}$, which from Eqs. (23), (27) and (33) can be seen to be given by $d^{2} R / d \hat{t}^{2}=-\left.a^{-2} \Phi_{N, \hat{x}}\right|_{\hat{X}}$. As in the case of static backgrounds, the expansion of the global lattice is therefore always decelerating (in terms of the proper distance, $R$ ), as long as the force from the Newtonian potential is attractive.

\section{Relating Time-dependent \& Time-independent Descriptions}

One could now ask if it is possible to remove the vacuum potentials by transforming the $\hat{X}=\hat{X}(\hat{y}, \hat{z})$ solutions above to different homogeneous and isotropic backgrounds. We find that this is indeed possible under the coordinate redefinitions

$$
\begin{aligned}
& \hat{t}=t-\frac{a_{, t}(t)}{a(t)} \frac{\left(x^{2}+y^{2}+z^{2}\right)}{2}+O(3) \\
& \hat{x}=\frac{x}{a(t)}\left[1-\left(\frac{c_{1}}{6 a^{3}(t)}+\frac{k}{4 a^{2}(t)}\right)\left(x^{2}+y^{2}+z^{2}\right)\right]+O(4) \\
& \hat{y}=\frac{y}{a(t)}\left[1-\left(\frac{c_{1}}{6 a^{3}(t)}+\frac{k}{4 a^{2}(t)}\right)\left(x^{2}+y^{2}+z^{2}\right)\right]+O(4) \\
& \hat{z}=\frac{z}{a(t)}\left[1-\left(\frac{c_{1}}{6 a^{3}(t)}+\frac{k}{4 a^{2}(t)}\right)\left(x^{2}+y^{2}+z^{2}\right)\right]+O(4) .
\end{aligned}
$$

The argument of $a$ is made explicit here, as it is now the case that $a(\hat{t}) \neq a(t)$. Instead we have to Taylor expand to find

$$
a(\hat{t})=a(t)\left(1-\frac{a_{, t}^{2}(t)}{a(t)} \frac{\left(x^{2}+y^{2}+z^{2}\right)}{2}\right)+O(4) .
$$

The line-element that results is then the static one specified in Eq. (10), with

$$
\phi=\frac{\Phi_{N}}{a(\hat{t})}+O(4) .
$$


We can then see that a boundary at $\hat{X}=\hat{X}_{0}\left(1-\left(\hat{y}^{2}+\hat{z}^{2}\right) k / 4\right)+O(4)$, in the coordinates of Eq. (22), is equivalent to the solution given in Eq. (21) with

$$
X_{c}(t)=a(t) \hat{X}_{0}\left(1+\frac{k \hat{X}_{0}^{2}}{4}\right)+O(4) .
$$

The constant $k$ then determines the asymptotic form of $X_{c}(t)$ in the usual way that is familiar from the solutions to the Friedmann equation.

We have now shown that one can describe the space-time geometry inside each of our cells as either linearly perturbed FRW, as in Eq. (22), or as linearly perturbed Minkowski space, as in Eq. (10), and that these two descriptions are equivalent to each other up to a coordinate transformation. One could argue, however, that the more natural description is in terms of the static coordinate system of Eq. (10). In these coordinates the perturbations can be consistently described as being solely due to Newtonian potentials. This is not true in the FRW coordinates, where an additional potential of the form $\Phi \sim r^{2}$ is also required within each cell (unless one wants to use an open, empty Milne universe as the background cosmology). If one were to choose to use the FRW description, it would therefore seem necessary to understand the effects that these potentials have on observable quantities, which are discussed further in Section 9 , Using the Newtonian (static) description, for calculations of luminosity distances etc., however, requires taking into account boundary condition between the different regions in which Newtonian descriptions are internally applicable. This will also be discussed in Section 9 ,

\section{Comparison with Perfect Fluid FRW Cosmology}

As discussed in Section 2, it has been suggested that the formation of structure in the Universe could lead to modifications of the expected FRW cosmological expansion, and in particular the scale of expansion. We will now use the model described in the previous section to address this issue. As already shown, the functional form of the constraint and evolution equations are the same as the Friedmann equations of FRW. This does not, however, guarantee that the solutions of these equations are identical to FRW solutions. In particular, we need to verify that the energy density corresponding to a given expansion rate is the same as expected from FRW, and that in spatially curved solutions the scale of expansion is also as expected.

\subsection{Spatially Flat Cosmologies}

First of all let us consider a spatially flat model. In this case $a(t)$ is scale-invariant, and so we only need to check that the value of the expansion rate for a given energy density is the same as prescibed by the usual Friedmann equation. To do this, we will find numerical solutions for the potentials $\phi$ and $\Phi$ that satisfy

$$
\nabla^{2} \phi=\hat{\nabla}^{2} \Phi-\frac{c_{1}}{a}=O(4),
$$




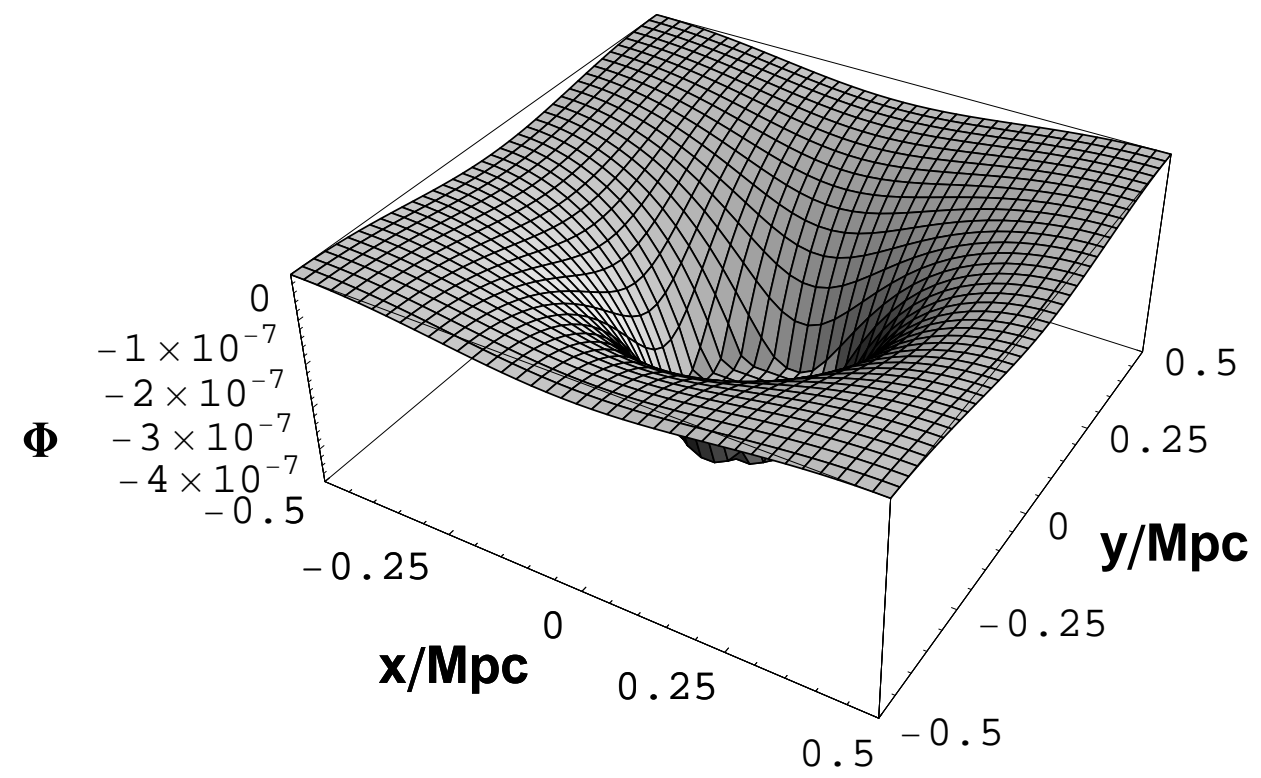

Figure 3. The potential $\Phi$ satisfying the field equation 42), and the boundary conditions (43) with a central mass of approximately Milky Way size, in a 1Mpc sized cubic cell, and in the plane $z=0$.

with boundary conditions given by

$$
\left.(n \cdot \nabla \phi)\right|_{X}+X_{\mid A A}=\left.\hat{n} \cdot \hat{\nabla} \Phi\right|_{\hat{X}}=O(4),
$$

and with a singularity at the centre of the cell. This solution is shown in Figure 3, for a central mass approximately as massive as the Milky Way, in a cubic cell of width $1 \mathrm{Mpc}$. We can then verify that, for fields satisfying Eqs. (42) and (43), we have

$$
\begin{aligned}
& \left.(n \cdot \nabla \phi)\right|_{X} \simeq-\frac{4 \pi}{3} \rho X, \\
& \hat{\nabla}^{2} \Phi \simeq-4 \pi a^{2} \rho,
\end{aligned}
$$

where $\rho=m / V, m$ is the central mass, and $V$ is the spatial volume of the cell (in either coordinate system, to the required accuracy). The $\simeq$ sign here means equal up to terms of $O(4)$. Substituting Eq. (44) into (16), or Eq. (45) into (23), we then recover the usual Friedmann equation, up to corrective terms of $O(4)$. The expansion rate for a given density is therefore the same as in a perfect FRW universe, to the required order. This result can also be obtained from Gauss's theorem by noting that from Eqs. (31) and (32) we have

$$
\begin{aligned}
\frac{1}{a^{2}} \int_{V} \hat{\nabla}^{2} \Phi d V & =\frac{1}{a^{2}} \oint \hat{n} \cdot(\hat{\nabla} \Phi) d S=O(4), \\
& =\frac{c_{1} V}{a^{3}}+4 \pi m+O(4)
\end{aligned}
$$

where integrals are performed over the volume of a cell, $V$, enclosed by the surface, $S$, and $m$ is the mass of a central singularity. It is then clear that $c_{1}=-4 \pi \rho a^{3}+O(4)$, which on substitution into Eq. (23) again gives the usual Friedmann equation, up to terms of $O(4)$. 
Finally, we point out that the $\rho$ occurring in Eqs. (44) and (45) does not correspond to a local energy density, and hence that these equations should not be considered as actual field equations themselves (as they are in studies of perturbed FRW solutions, with perfect fluids), but rather as a derived consequence of the field equations. What has then be shown above is that the large-scale evolution of a spatially flat universe can be recovered, up to some prescribed level of accuracy, even when the matter content is arbitrarily inhomogeneous (at least, when the matter is arranged in a regular way). This result relies only on the validity of local perturbative expansions about Minkowski space in the vicinity of isolated masses, which is not often considered an ambiguous procedure. This is not the same thing as taking for granted the validity of the usual perturbed FRW approach and showing that regions of space-time can then be described locally as perturbed Minkowski space. In that case the large-scale expansion is given by the assumed global background, rather than as a consequence of any boundary conditions.

\subsection{Non-flat Cosmologies}

Now let us consider models with non-zero spatial curvature. In these cases the corrections to Eqs. (42) and (43) due to the curvature are of $O(4)$ only, and hence do not affect our numerical calculation of $\phi$. Furthermore, the RHS of Eqs. (44) and (45) are also only corrected by $O(4)$ terms. It is therefore the case the energy density in the spatially curved models is also the same as in the usual FRW Friedmann equations, up to the required order of accuracy. It now remains to confirm this is also true for the spatial curvature terms in Eqs. (16) and (23).

In order to compare the scale of expansion in the models we have been considering to the usual FRW perfect fluid solutions we need to know if the $k$ appearing in Eq. (23) is the same as $K$, which determines the global spatial curvature of the homogeneous FRW solutions. Global curvature in the lattice models has not yet been shown to be equal to $k$, which so far only describes spatial curvature inside of each cell in the coordinates used in Eq. (22), and the position of the boundaries via Eq. (30).

Global curvature in the lattice models should be inferred using the angle at which the different faces of a single cell meet. To see this, consider a lattice made from cells that are a single repeated regular polytope. the resulting structure is then known as a polychoron, and there are 6 different convex polychora with which we could model a lattice with positive spatial curvature [18]. Now, take as an example the largest of these configuration, which contains 600 simplexes, and is known as a hexacosichoron. It has 5 simplexes meeting around each edge of every face of every cell, and so in order to create such a structure we would need the angle with which the cell boundaries described by Eq. (30) meet to be $360^{\circ} / 5=72^{\circ}$, in a hyper-surface orthogonal to the world-line of a time-like observer on the edge of the cell face. This angle can be seen to depend on the value of $k$ in Eq. (30), and once it has been achieved the global curvature of the lattice is set by the curvature $K$ of the hyper-sphere with an image of the same polychoron on 
its surface volume.

We can now note that the boundary positions of each cell, when considered as an image on a global hyper-sphere, should be described by geodesics of that hyper-sphere, due to the symmetry about each boundary. Ensuring that Eq. (30) describes a geodesic in such a space therefore allows us to compare $k$, from the boundary position equation, with $K$ from the geodesic equations on the hyper-sphere. To do this, we differentiate Eq. (30) twice with respect to some affine parameter, $\lambda$, along a curve in the boundary, giving

$$
\ddot{X}=-\frac{k}{2} X_{0}\left(\dot{y}^{2}+\dot{z}^{2}+y \ddot{y}+z \ddot{z}\right)+O(4),
$$

where over-dots here denote differentiation with respect to $\lambda$. Using the metric of a hyper-sphere with spatial curvature $K$,

$$
d s^{2}=\frac{d x^{2}+d y^{2}+d z^{2}}{\left(1+\frac{K}{4}\left(x^{2}+y^{2}+z^{2}\right)\right)^{2}},
$$

we then find that in order for $\ddot{X}, \ddot{y}$ and $\ddot{z}$ to describe a geodesic we must have

$$
\begin{aligned}
& \ddot{X}=-\frac{K X}{2}\left(\dot{y}^{2}+\dot{z}^{2}\right)+O(4), \\
& \ddot{y}=\frac{K y}{2}\left(\dot{y}^{2}-\dot{z}^{2}\right)+K z \dot{y} \dot{z}+O(4) \\
& \ddot{z}=\frac{K z}{2}\left(\dot{z}^{2}-\dot{y}^{2}\right)+K y \dot{y} \dot{z}+O(4),
\end{aligned}
$$

where we have used the result $X_{\mid A} \sim O(1)$ to assign $\dot{X}$ an $O(1)$ of smallness, compared to $\dot{y}$ and $\dot{z}$. Substituting these expressions into Eq. (48) then gives

$$
k=K+O(4) .
$$

We have therefore shown that the boundaries described by Eq. (30) are, in fact, geodesics of the hyper-sphere given in Eq. (49) when Eq. (53) is satisfied. The global curvature of lattice must therefore be given by $k$, with only corrections up to $O(4)$ allowed.

With the energy density and spatial curvature terms on the RHS of Eq. (23) being equal to their values in the corresponding FRW solutions, the scale of expansion of the lattice model must therefore also be equal to that of the FRW solutions. We have now shown that the large-scale dynamics of these models are indistinguishable from those of perfect fluid FRW solutions with the same global energy density and spatial curvature, up to the required accuracy. As we have also shown that the geometry inside each cell can be described as perturbed FRW geometry, it then follows that the background FRW solution of each cell can also be taken to be the global solution. We have therefore shown that a global perturbed FRW space-time geometry is sufficient to describe the situation of n regularly spaced discrete masses, with no corrections beyond $O(4)$ required (unless one wants to describe the region close to a compact object, or the early universe). 


\section{Observables in a Lattice}

We have so far considered the space-time geometry of a universe composed of a large number of discrete objects, nearby to each of which post-Newtonian gravity is a good approximation. The large-scale evolution of the global space-time has then been deduced through the applications of Israel junction conditions between the different local patches, and it has been found that the usual linearly perturbed FRW cosmology is still a good approximation to the space-time geometry (as long as one does not approach a singularity) even though the density fluctuations are arbitrarily large. These results are promising evidence for the applicability of perturbed FRW cosmology to at least some situations in which the density contrast is large. It does not, however, guarantee that cosmological observations in these space-times will be similar to those made in a perfect FRW space-time. We will consider this problem in the present section.

There are, of course, a great number of studies on observable quantities such as redshift and luminosity distance in inhomogeneous cosmological models. This is particularly true in the case of perturbed FRW cosmology, where the relevant formalism was first given by Kristian and Sachs [19]. With regards to other approaches, the 'Swiss cheese' models, in which Einstein-Strauss vacuoles [5] or spherical Lemaître-TolmanBondi patches [20]-[22], are embedded in a perfect FRW background have also been well studied [23]-[29]. Such studies allow for the contribution of non-linear and nonperturbative effects, but only for spherically symmetric inhomogeneities with perfect FRW boundary conditions. Observables in non-FRW models with regularly spaced discrete masses were studied in [7] and [8], using the Lindquist-Wheeler model.

Here one could proceed with calculating redshifts and luminosity distances in at least two different ways: (i) Within the context of the perturbed FRW geometry given by Eq. (22), or (ii) in terms of the static geometry given by Eq. (10). In either case the results should be the same, and the first step is to calculate the photon trajectories within each cell. These should satisfy the geodesic and null constraint equations

$$
k^{a} k_{; a}^{b}=0 \quad \text { and } \quad k^{a} k_{a}=0,
$$

where $k^{a}$ is the 4 -vector tangent to the null geodesics. In either of the two cases mentioned above one must then deal with the boundary conditions between cells, in order to propagate photons over cosmologically relevant distance scales. This should proceed as follows: One should define a congruence of time-like geodesics that are comoving with the boundary of the first cell, which can be labelled $u^{a_{1}}$. One can then decompose $k^{a_{1}}$ into components tangential and orthogonal to $u^{a_{1}}$ as

$$
k^{a_{1}}=\left(-u^{b_{1}} k_{b_{1}}\right)\left(u^{a_{1}}+n^{a_{1}}\right),
$$

where $n^{a_{1}} u_{a_{1}}=0$, and $n^{a_{1}} n_{a_{1}}=1$. The frequency and direction of a photon that passes by an observer on the boundary are then given by $-u^{a_{1}} k_{a_{1}}$ and $n^{a_{1}}$, respectively. Here we have used subscript 1 on space-time indices to denote that these quantities are being evaluated in the coordinates used in the first cell. 
Now consider an observer on the boundary of the second cell following a curve in the time-like congruence $u^{a_{2}}$, into which the photon is propagating. A similar decomposition to Eq. (55) can be performed, and the frequency and direction of the photon measured by this observer are given by $-u^{a_{2}} k_{a_{2}}$ and $n^{a_{2}}$. As the observer on the boundary of the first cell is to be identified with the observer on the boundary of the second cell, when the junction conditions are applied, the frequency and direction of photons measured by the two observers should be the same. The quantities $-u^{a_{2}} k_{a_{2}}$ and $n^{a_{2}}$ are therefore given by

$$
\begin{aligned}
& -u^{a_{2}} k_{a_{2}}=-u^{a_{1}} k_{a_{1}} \\
& n^{a_{2}}=\frac{\partial x^{a_{2}}}{\partial x^{a_{1}}} n^{a_{1}},
\end{aligned}
$$

where $\left(\partial x^{a_{2}} / \partial x^{a_{1}}\right)$ denotes the relevant transformation between the two coordinate systems. These four equations provide enough information to calculate the four components of $k^{a_{2}}$, when $k^{a_{1}}$ is known. We therefore have the initial conditions we need to propagate the congruence of null geodesics through the second cell, where the same procedure as just described can be applied again and again to propagate through large numbers of cells.

With a knowledge of the 4 -vector $k^{a}$ we can now calculate cosmological redshifts and luminosity distances in the model under consideration. The first of these quantities is given by the expression

$$
1+z=\frac{\left.\left(-u^{a} k_{a}\right)\right|_{e}}{\left.\left(-u^{b} k_{b}\right)\right|_{o}},
$$

where, as already explained, $-u^{a} k_{a}$ corresponds to the frequency of a photon measured by an observer following a curve in $u^{a}$, and we have used subscripts $o$ and $e$ to denote the points on the null curve where observation and emission occur. There is some ambiguity here in exactly how one chooses the congruences $u^{a}$, as the space-time is a vacuum outside of the central object of each cell, and so no preferred set of curves given by a background fluid can be assumed. As long as one is consistent in how such congruences are chosen from cell to cell, however, the differences should be small between the different possible choices, as long as the relative velocity between two observers following the curves in the two congruences is also small.

Finally, in order to obtain luminosity distances, one needs to integrate the Sachs optical equations along the null curves that were found above. These are:

$$
\begin{aligned}
& \frac{d \theta}{d \lambda}+\theta^{2}-\omega^{2}+\sigma^{*} \sigma=-\frac{1}{2} R_{a b} k^{a} k^{b} \\
& \frac{d \omega}{d \lambda}+2 \omega \theta=0 \\
& \frac{d \sigma}{d \lambda}+2 \sigma \theta=C_{a b c d}\left(t^{*}\right)^{a} k^{b}\left(t^{*}\right)^{c} k^{d},
\end{aligned}
$$

where $\theta, \omega$ and $\sigma$ are the expansion, rotation and complex shear scalars, respectively, $\lambda$ is an affine parameter along the curve, and $t^{a}$ is a complex vector field obeying $t^{a} k_{a}=0$, $t^{a} t_{a}=0$ and $t^{a}\left(t^{*}\right)_{a}=1$. Here, $R_{a b}$ is the Ricci tensor and $C_{a b c d}$ is the Weyl tensor. In 
propagating photon trajectories between cells one should transform $t^{a}$ in a similar way to $k^{a}$. As always, once the $\theta, \omega$ and $\sigma$ are known along the null trajectories, then the angular diameter distances can be calculated by $r_{A} \propto \exp \left\{\int_{\mathrm{e}}^{\mathrm{o}} \theta \mathrm{d} \lambda\right\}$, and the luminosity distances by $r_{L}=(1+z)^{2} r_{A}$.

We will now make some comments about the procedure outlined above, which will not be performed explicitly here. Firstly, we note that in perfect fluid FRW cosmology the RHS of Eq. (59) is non-zero and the RHS of Eq. (61) is zero, while in the present situation the exact opposite is true: The RHS of Eq. (59) is zero and the RHS of Eq. (61) is non-zero. This appears to have been first noticed by Bertotti in [30]. Now, while the Ricci curvature source term is switched off in the present case, the $\sigma^{*} \sigma$ term in Eq. (59) becomes non-zero, due to the Weyl term on the RHS of Eq. (61) being non-zero. It has been argued by Weinberg that the effect of the non-zero shear in Eq. (59) should entirely replace the missing Ricci curvature term, so that the luminosity distance is, in fact, unaffected by the matter being clumped [32. This argument is essentially based on conservation of photon number, but has since been shown to be questionable [33]. In particular, Weinberg's argument neglects the occurrence of caustics in the congruence of null geodesics along which we observe. Caustics should be expected to occur when shear is allowed to be non-zero, as it necessarily has to be here. Furthermore, Weinberg's argument relies on spatial areas taking the same value in clumpy cosmological models as they do in FRW ones, and on being able to make large numbers of observations over the entire sky. With finite observations made at relatively low redshifts $(z \lesssim 1)$, shear has in fact been shown, for at least some inhomogeneous cosmological models, to remain relatively low along typical geodesics [7, 34]. We expect this to be true in the present situation as well, so that $\theta \simeq 1 / \lambda$ when $z \lesssim 1$. The neglect of shear in this way is sometimes known as the Dyer-Roeder approximation [35], and while it is expected to be a good approximation for most situations at low redshifts, it may not be so at high redshifts. The effect that shear can have on the CMB sky has been discussed in [33], where the consequences of caustics in particular are considered.

It now remains to consider redshifts along null geodesics. These are usually taken as being prescribed by the ratio of scale factors at different points on a curve, as in FRW cosmology, although this does not necessarily have to be the case in inhomogeneous models, and in general one should calculate it using Eq. (58). In the Lindquist-Wheeler model it was recently found that a non-negligible deviation from the FRW value of redshift can occur [7]. For the model being considered here, we do not expect a repeat of this result. The reason for this is that we can choose the time-like congruence $u^{a}$ to be that of an observer at fixed spatial position, in the coordinates of Eq. (22). Observers following such a congruence will not be geodesic, but this does not matter, and it should only be a small correction if one wishes to consider geodesic observers, as peculiar velocities in the coordinates of Eq. (22) are all $O(1)$ small. Now, if the 4-vector tangent to the null geodesics, $k^{a}$, were the same as in FRW then we would expect exactly the same redshift along each curve. Here we should expect small perturbations to this field, so the actual redshift should also be corrected. Assuming the photon trajectories 
do not pass too close to the Schwarzschild radius of any central masses, however, these corrections are expected to be small, as is the case in Swiss cheese models [23]. We therefore do not expect large deviations from the expected FRW results on redshift, as were found in [7], although one would need to perform the explicit numerical integration described above to be sure.

The picture we have outlined here is quite similar to that used by Holz and Wald in their "new method for determining cumulative gravitational lensing effects" [36]. In this work the authors use Newtonian potentials to calculate the shear and expansion of a bundle of null geodesics as it passes through a region, updating the redshift using FRW relations as they leave one region and enter the next. This is just the situation here: Inside each cell the space-time can be well described by the perturbed Minkowski space of Eq. (10), and by propagating geodesics between cells one should pick up a similar redshift to that expected from FRW cosmology (up to possible effects caused by perturbations to $k^{a}$, as just discussed).

\section{Discussion}

In summary, we have considered n-body cosmological models that do not require any averaging procedures. These models have been constructed using a lattice made from a large number of symmetric cells, each containing identical central masses. By applying appropriate junction conditions between cells we then find a set of evolution equations that specify the motion of the cell boundaries, and hence the global evolution of the space-time. In all of the cases we have considered we find that the cell boundaries must be in free fall, and decelerating in their expansion away from the cell centres. We find that the space-time geometry inside each cell can be described as fluctuations around either static or time-dependent FRW backgrounds. In order to apply the FRW description one must include potentials of the form $\Phi \sim r^{2}$, whose gradients must be of the same order of magnitude as the Newtonian potentials that are also present. Treating the space-time inside each cell as fluctuations around Minkowski space, however, requires Newtonian potentials only. One may then consider perturbed Minkowski space to be a more natural local description of the space-time.

We have compared the resulting large-scale dynamics of the cosmological model under consideration with those of a perfect fluid FRW cosmology. We find that for spatially flat universes the expansion rate for a given energy density has just the expected FRW value. Furthermore, we have compared the scale of expansion of non-flat models with spatially curved perfect fluid FRW universes and found that they are also the same, up to the required accuracy. We emphasize that this result does not follow directly from the fact that the space-time geometry inside each cell can be written as perturbed FRW: It could, in principle, have been the case that the scale of curvature of the global lattice was different from the scale inferred from the relevant FRW background used inside each cell (in fact, from a number of the previous studies discussed in Section 2, it would seem that one may have expected such a result). Instead, what we find here is that 
discretisation of the matter content of the Universe does not have to affect the global background rate of expansion.

These results are a consequence of explicitly solving boundary conditions between different regions that can, individually, be described as perturbations around highly symmetric backgrounds. That such a description is possible within each cell does not, however, automatically mean that one can treat the entire Universe as fluctuations around a single universal FRW background. Such a result has to be shown to be true by reconstructing the global geometry from the local geometry that is appropriate within each of these regions. This is what we have done here, finding that for the simple case of regularly spaced masses a single global FRW background with small perturbations around it is a perfectly adequately description of the entire space-time. That a global FRW background is a valid description in the present case, however, does not automatically mean that this will also be true for irregular arrangements of massive objects. Such a result remains to be shown.

As already discussed, the model we have considered here can be interpreted solely in terms of perturbations about Minkowski spaces (albeit a different Minkowski space within each cell). That this is possible gives one greater reason to expect our perturbative expansion to be valid in the regime of non-linear density contrasts than is the case in globally FRW perfect fluid cosmology. As discussed in [3], there are terms at higher orders in the latter case that one may expect to blow up when $\delta \rho / \rho$ becomes large. With the former case of Newtonian and post-Newtonian fluctuations about Minkowski space, however, the situation is different. The terms that could potentially blow up are absent, and hence we have greater confidence in the applicability of the post-Newtonian description of these systems when density contrasts are large.

To determine whether or not the results we have found here carry over to more realistic models of the Universe, where masses are irregularly spaced, and dispersed matter is also present, will require further study, and more refined models. However, we do expect our results to be of use for constraining the possible effects that structure formation could have on the background expansion of the Universe, as well as for testing the viability of mechanisms that have been constructed in order to correct for backreaction and averaging: If corrections are predicted in situations where it is known that none occur, then the frame-work within which they have been identified should be questioned. 


\section{Acknowledgements}

I am grateful for valuable discussions with Pedro Ferreira, Chris Clarkson, George

Ellis and Thomas Sotiriou. I also wish to acknowledge the support of Jesus College Oxford, the BIPAC, and CERN.

\section{References}

[1] Ellis G F R 1984 in General Relativity and Gravitation, Ed. Bertotti B et al. Reidel, Amsterdam

[2] Buchert T 2000 Gen. Rel. Grav. 32105

[3] Räsänen S 2010 Phys. Rev. D 81103512

[4] McVittie G C 1931 Mon. Not. Roy. Astron. Soc. 91274

[5] Einstein A and Strauss E G 1945 Rev. Mod. Phys. 17120

[6] Lindquist R W and Wheeler J A 1957 Rev. Mod. Phys. 29432

[7] Clifton T and Ferreira P G 2009 Phys. Rev. D 80103503

[8] Clifton T and Ferreira P G 2009 JCAP 1026

[9] Uzan J-P, Ellis G F R and Larena J 2010 arXiv:1005.1809[gr-qc]

[10] Zalaletdinov R M 1997 Bull. Astron. Soc. India 25401

[11] Coley A A, Pelavas N and Zalaletdinov R M 2005 Phys. Rev. Lett. 95, 151102

[12] Buchert T 2008 Gen. Rel. Grav. 40467

[13] Buchert T, Larena J and Alimi J-M 2006 Class. Quant. Grav. 236379

[14] Israel W 1966 Nuovo Cim. B 441

[15] Israel W 1967 Nuovo Cim. B 48463

[16] Will C M 1993 Theory and Experiment in Gravitational Physics, revised edition, CUP

[17] Saari D G 1971 Trans. Am. Math. Soc. 156219

[18] Coxeter H S M 1973 Regular Polytopes, 3rd. ed., Dover Publications

[19] Kristian J and Sachs R K 1966 Astrophys. J., 143379

[20] Lemaître G 1933 Ann. Soc. Sci. Brussels A $\mathbf{5 3} 51$

[21] Tolman R C 1934 Proc. Nat. Acad. Sci. USA 20169

[22] Bondi H 1947 Mon. Not. Roy. Astron. Soc. 107410

[23] Kantowski R 1969 Astrophys. J. 15589

[24] Marra V, Kolb E W, Matarrese S and Riotto A 2007 Phys. Rev. D 76123004

[25] Biswas T, Mansouri R and Notari A 2007 JCAP 0712017

[26] Biswas T and Notari A 2008 JCAP 0806021

[27] Brouzakis N, Tetradis N and Tzavara 2007 JCAP 0702013

[28] Brouzakis N, Tetradis N and Tzavara 2008 JCAP 0804008

[29] Clifton T and Zuntz J 2009 Mon. Not. Roy. Astron. Soc. 4002185

[30] Bertotti B 1966 Proc. Roy. Soc. Lond. A 294, 195

[31] Etherington I M H 1933 Phil. Mag. ser. 7 15, 761

[32] Weinberg S 1976 Astrophys. J. 16857

[33] Ellis G F R, Bassett B A C C and Dunsby P K S 1998 Class. Quant. Grav. 152345

[34] Kainulainen K and Marra V 2009 Phys. Rev. D 80123020

[35] Dyer CC and Roeder R C 1973 Astrophys. J. Lett. 180 L31

[36] Holz D E and Wald R M 1998 Phys. Rev. D 58063501 